\title{
Review
}

\section{The Generality of Strength Adaptation}

\author{
Samuel L. Buckner, Tayla E. Kuehne, Noam Yitzchaki, Wenyuan G. Zhu, \\ Megan N. Humphries, Jeremy P. Loenneke
}

The generality of strength suggests that a "strong" individual will typically exhibit higher values of strength across a wide range of strength tasks for a given muscle relative to their weaker counterpart. This concept is often extended to adaptation, suggesting that increasing strength on a given movement or strength task with a given muscle should reflect an increase in other movements or tasks using that same muscle. The concept of a generality of strength adaptation appears less supported in the literature.

Objective: To elaborate on recommendations for strength assessment, providing a focus on the "generality of strength" and the "generality of strength adaptation."

Design \& Methods: We reviewed the literature on a generality of strength. In addition, we examined the resistance training literature to provide evidence and discussion on a generality of strength adaptation.

Results/Conclusions: The generality of strength adaptation, even across strength skills using the same muscle on related movements seems quite low. Although some studies show a weak generality of strength adaptation and others show no generality of strength adaptation, it appears that increases in strength diminish as the strength assessment becomes farther removed from the actual training stimulus.

(Journal of Trainology 2019;8:5-8)

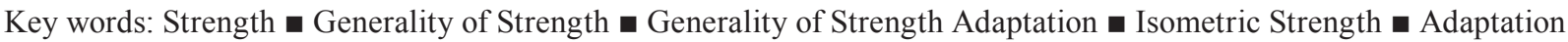

\section{STRENGTH ADAPTATION TO RESISTANCE EXERCISE}

Strength adaptations observed following resistance training appear largely dictated by the principle of specificity. ${ }^{1}$ In this light, it seems reasonable to suggest that neural mechanisms, and perhaps other local factors are driving increases in strength. ${ }^{2}$ Such factors may include changes in the pattern of calcium release ${ }^{3}$, changes in the components involved in the excitation contraction coupling process ${ }^{4}$, and changes in the composition of myosin motors 5 . Muscle hypertrophy remains a candidate for strength adaptation; however, in many situations it has been suggested that hypertrophy plays a negligible role. ${ }^{6,7}$ Increases in strength following a resistance training program are often measured using several assessments, some of which may be considered specific to the training stimulus, whilst others appeal less to specificity (i.e., involving the same muscle groups but different contraction type/movement). It has been suggested that multiple strength measures may better reflect strength adaptations acquired from a resistance training program. ${ }^{8}$ However, given the different magnitudes of change observed amongst different strength tests ${ }^{9}$, it seems likely that these various assessments are providing different information. The purpose of this paper is to elaborate on recommendations for strength assessment, providing a focus on the "generality of strength" and the "generality of strength adaptation."

When measuring the strength of a muscle, a "strong" indi- vidual will typically exhibit higher values of strength across a wide range of strength tasks for a given muscle relative to their weaker counterpart. For example, Hortobagyi et al. ${ }^{10}$ compared strength across isokinetic, hydraulic and one repetition maximum (1RM) free weight bench press exercises finding that, although values across tests were remarkably different, there were strong positive associations amongst the four separate tests of bench press $(r \geq 0.80)$. Meaning, individuals who tested higher on one task tended to also test higher on other related tasks using the same muscles. This phenomenon is known as the "generality of strength," which suggests that "if strength is measured by different contraction modes and velocities, the same relative ranking of an individual's performance would be obtained on the different tests." 10

A generality of strength was demonstrated as early as 1957 when Rasch ${ }^{11}$ showed that at a single time point, 24 college aged males had a mean isometric elbow flexion strength of 43.2 (SD 3.4) lbs. and a mean isotonic elbow flexion strength of 41.8 (SD 6.9) lbs. Similarly, Rasch and Pearson ${ }^{12}$ found that, in a group of untrained college men, mean isometric strength and "breaking point" strength were similar in the muscles of the elbow flexors $(49.5 \mathrm{lbs}$. and $50.9 \mathrm{lbs}$. respectively). Thus, in some cases it is possible to measure similar strength values using different strength tasks at a single timepoint. Given the fairly strong "generality of strength" when comparing individuals at a single time point, adaptations to resistance exercise are often assessed using a single strength

Received February 24, 2019; accepted April 28, 2019

From the USF Muscle Lab, Exercise Science Program, University of South Florida, Tampa, FL (S.L.B., T.E.K., N.Y., W.Z., M.H.) and Department of Health, Exercise Science, and Recreation Management. Kevser Ermin Applied Physiology Laboratory, The University of Mississippi, University, MS (J. P.L.)

Communicated by Takashi Abe, $\mathrm{PhD}$

Correspondence to: Dr. Samuel L. Buckner, University of South Florida, 4202 E. Fowler Ave. PED 214, Tampa, Florida, 33620-8600, USA. Email: slbuckner@usf.edu

Journal of Trainology 2019;8:5-8 @2012 The Active Aging Research Center http://trainology.org/ 
assessment (e.g., isotonic 1RM strength) or even multiple (e.g., isometric, isotonic and/or isokinetic) assessments which are then believed to provide information regarding "general strength" of a muscle. This concept underlies much of strength and conditioning, which seeks to increase strength on various tasks in the weight room and facilitate performance benefits on different sport related activities and movements. This assumes, however, that the generality of strength extends itself into adaptation (i.e., a generality of strength adaptation). A generality of strength adaptation would suggest that increasing strength on a given movement or strength task with a given muscle should reflect an increase in other movements or tasks using that same muscle. The concept of a generality of strength adaptation is rarely discussed, yet is often assumed to exist. Although it has received little attention in the literature, the current practices of strength assessment following resistance training (e.g., using multiple strength tests) has inadvertently demonstrated the generality of strength adaptation.

\section{HOW STRENGTH IS MEASURED}

The force producing capabilities of a muscle are often measured using several different assessments. Multiple tests are employed to account for the fact that a single strength assessment (e.g., isotonic strength) may not reflect all strength adaptations acquired following a resistance training program. ${ }^{8}$ Although the use of several strength assessments is thought to provide a more global picture of strength adaptation, it must be acknowledged that changes in strength on assessments further removed from the training style (e.g., if you train isotonically and test strength isometrically) are of a lesser magnitude compared to a strength assessment that appeals to specificity of the training program (i.e., isotonic testing for an isotonic training program). It seems logical that strength increases on tasks/contraction modes different from the training are more likely indicative of a "generality of strength adaptation; " however, little work has been conducted to better understand the meaningfulness of increases in general strength.

Highly influential in the understanding of strength adaptations have been the emergence of different loading paradigms within the resistance training literature (e.g., high loads vs. low loads). This literature suggests that high load training produces similar muscle growth as low load training, despite more robust increases in isotonic 1RM strength. ${ }^{13,14}$ However, when strength is measured using a test to which both groups are "naive" (e.g. isometric), differences in strength are often less apparent. ${ }^{13,15-18}$ The strength differences observed between loading schemes has further enforced the tendency to measure strength adaptations using multiple different assessments. ${ }^{8}$ In these instances, isotonic 1RM strength assessment appeals to specificity (load and movement) for the high load training condition, while appealing less to specificity (movement only) for low load training alternatives. It has been suggested that these strength differences can be minimized if additional strength assessments are included. Interestingly, the motivation for including these assessments has largely been to capture the strength adaptations expected to accompany the muscle growth observed. Under the model proposed by Moritani and deVries ${ }^{19}$, it is difficult to reconcile the differences in muscle strength between low load and high load training programs given similar levels of muscle growth. The assumption that growth must result in strength adaptation has led to interesting hypotheses, such as the "Theoretical Reverse Pattern of Adaptation." ${ }^{20}$ Herein, authors suggested that the smaller increases in strength following low load resistance training with blood flow restriction are a result of hypertrophy and that neural mechanisms will likely play a role as time progresses. Although this was a reasonable hypothesis at the time, it suggests that low loads will eventually (if given enough time) produce similar strength adaptations as high load resistance training, which does not seem likely given the lack of exposure to lifting heavy weight (i.e., specificity). ${ }^{13,14}$ Nevertheless, Morton et al. ${ }^{21}$ was able to demonstrate similar increases in the majority of $1 \mathrm{RM}$ strength assessments between high load and low load resistance training. Authors speculate that "maximal strength increases can be achieved with the use of either low or high loads, so long as there is periodic practice of lifting with heavier loads". ${ }^{21}$ The amount of practice necessary to augment strength may be dependent on the complexity of the skill as bench press strength increased more in the high load group compared to the low load group. Future studies incorporating low load training groups with and without periodic practice of lifting with heavier loads are necessary to better understand strength adaptations and amount of "practice" necessary to augment various strength outcomes. However, if low load training programs are not accompanied with some exposure to lifting heavy weight throughout the movement/skill of interest, it appears that maximal strength in that movement will only increase minimally (if at all). ${ }^{13,14}$

\section{THE GENERALITY OF STRENGTH ADAPTATION}

Rasch and colleagues succeeded in demonstrating that there exists a generality of strength: ${ }^{12,11}$ however, the same research group failed to show an equally strong generality of strength adaption following 6 weeks of training. ${ }^{1}$ Authors demonstrated that strength in the elbow flexors increased more when participants were tested in a position (erect vs. supine) and manner (dynamic vs. modified Martin technique) more similar to how they had trained. ${ }^{1}$ A lack of generality of strength adaptation has also been observed by Sale et al. ${ }^{22}$ who found that isotonic strength increased following 19 weeks of isotonic strength training, while conferring no increase in maximal isometric knee extensor strength. The authors anticipated increases in all measures of strength considering they had observed an increase in the cross-sectional area of the knee extensor muscles as measured by computed tomography. However, in light of their findings, the authors offered another mechanistic explanation for increased strength which relied primarily on neural adaptations. ${ }^{22}$ Similarly, Rutherford et al. ${ }^{23}$ found that maximum knee extensor strength increased robustly $(160 \%$ in males and $200 \%$ in 
females) following 12 weeks of knee extensor training, with much smaller changes observed in isometric torque $(20 \%$ in males, $3 \%$ in females). In addition to this, authors observed no significant changes in maximal oxygen uptake or maximal power output on a cycle ergometer. ${ }^{23}$ This suggests that making a muscle stronger isotonically may provide some benefit for isometric strength (i.e., generality of strength adaptation), but provide little benefit to other performance measures using the same muscle groups (i.e., maximal oxygen uptake or maximal power output on a cycle ergometer).

More recently, Fisher et al. ${ }^{24}$ provided some evidence of a generality of strength adaptation following 10 weeks of Romanian deadlift (RDL) training or isolated lumbar extension training. When examining increases in $1 \mathrm{RM}$ RDL strength, authors noted a larger increase in the group that performed RDL training [+23 (SD 9.65) kg] compared to the group that performed lumbar extension training [+10.2 (SD $9.88) \mathrm{kg}] .{ }^{24}$ Conversely, when examining changes in lumbar extension strength, only the lumbar extension training group demonstrated an increase. ${ }^{24}$ Insight into the generality of strength adaptation can also be gained from the low load training literature. Jessee et al. ${ }^{25}$ noted a $10 \%$ increase in isotonic knee extensor 1RM strength following isotonic training at moderate training load $(70 \% 1 \mathrm{RM})$. The study also included three very-low load training conditions (15\% 1RM with and without blood flow restriction) that observed no meaningful changes in isotonic knee extensor strength. However, when examining other tests of strength, the authors noted a main effect for time, with all groups increasing $4.8 \%$ in isometric knee extension strength and $6 \%$ in isokinetic strength at $180 \%$ sec. Interestingly, there was no statistically significant strength increase in isokinetic knee extension strength at $60 \%$ sec. Although strength differences between loading conditions (high load versus low load) were eliminated by examining tests that did not favor any given condition, it is possible that these small increase were simply an artifact of repeated strength assessment. Future studies could incorporate a nontraining control group that only performs testing to eliminate this possibility. In addition, these strength increases may be a result of the lower complexity (e.g., pulling a handle on a dynamometer in a single joint movement) of the overall assessment used. Nevertheless, if these groups were compared to another group that was training more specific to the additional strength tests (training isometric and testing isometric), it seems likely that the change would exceed that observed by conditions training isotonically.

Increases in non-specific strength are what we believe represent a generality of strength adaptation. The generality of strength adaptation, even across strength skills using the same muscle on related movements seems quite low. Figure 1

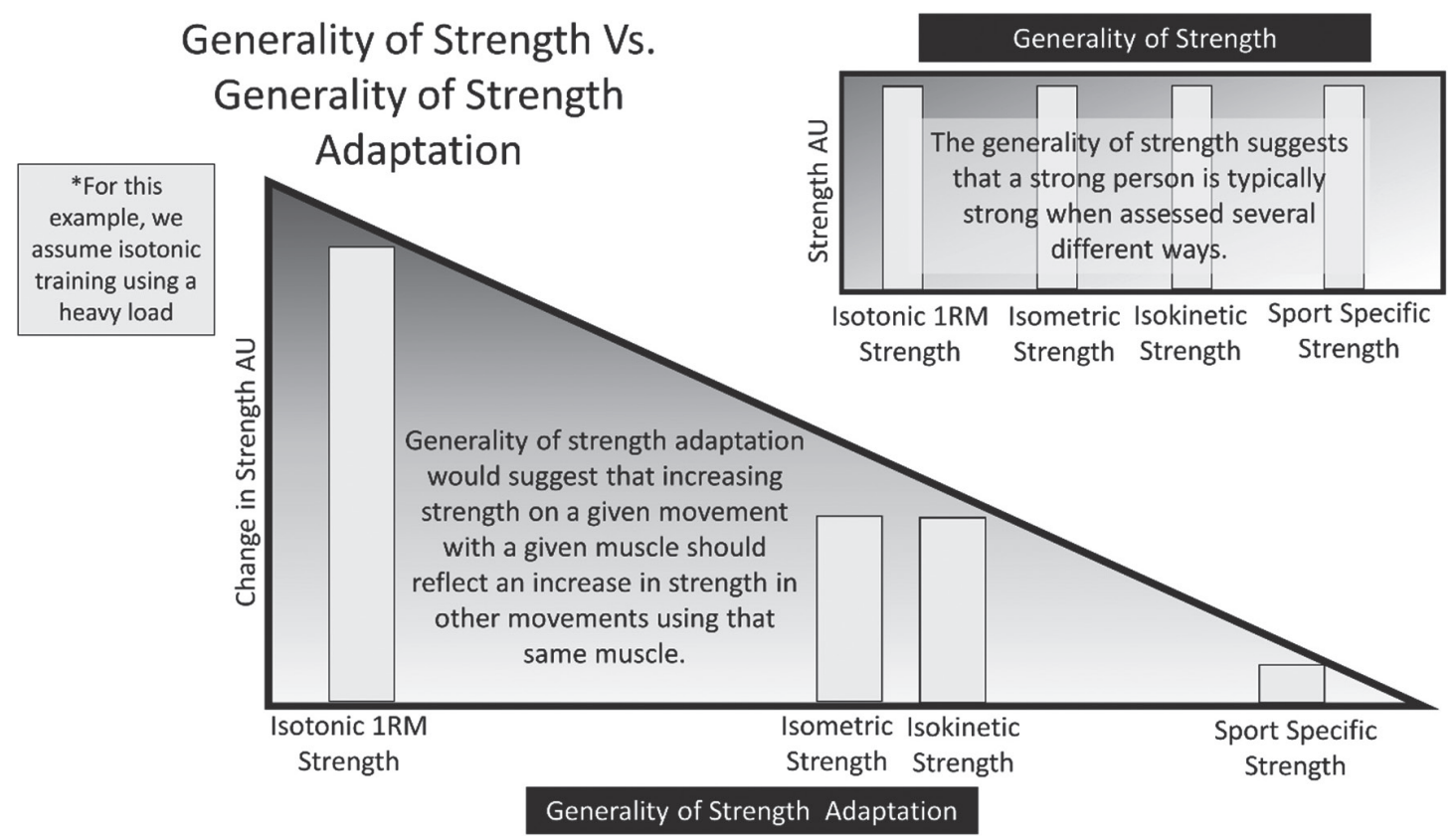

Figure 1

Figure 1 illustrates the hypothesized "Generality of strength" and the "Generality of strength adaptation." Although an individual's general strength rating may be high relative to their weaker counterpart across strength assessments (generality of strength), it appears that strength adaptations acquired from strength training are highly dependent on specificity (generality of strength adaptation). The further removed a strength assessment becomes from the actual training, the more difficult is to predict what adaptations will occur. However, the magnitude of change is expected to diminish as the skill/assessment becomes less specific to the training. We have included "sports specific strength" as this is often the most desired outcome, but also the farthest removed from training. 
provides an illustration of both the "Generality of Strength" (a) and the "Generality of Strength Adaptation (b). Although some studies show a weak generality of strength adaptation and others show no generality of strength adaptation, the figure conveys the diminishing benefits as the strength assessment becomes more removed from the actual training stimulus, which we hypothesize to be the case.

\section{CONCLUSIONS}

It has previously been suggested that multiple measures of strength be included in a resistance training program to better reflect the adaptations acquired by a training program, while minimizing the influence of specificity favoring one group over another. Although it seems worthwhile to still include multiple measures of strength, it is our opinion that multiple measures may simply provide information regarding how much "general strength" was acquired over the course of a training study. Thus, a test of isometric strength should not be used to demonstrate that specific strength adaptations are similar between high load and low loads training programs (as has been done in the past), but to demonstrate that a low load alternative can render a similar change in general strength as high load training, at least when tested on a simple movement for a given muscle. In addition, multiple measures of strength may inform us as to how much of a change in maximal strength through a given movement transfer to other movements using that same muscle. This may help to better inform strength and conditioning practice which appears to currently rely heavily on the "generality of strength" as opposed to the "generality of strength adaptation." When training a given muscle through a given movement, the most robust strength increases will be observed on the skill and movement that were specifically trained. Other tasks using that same muscle on different (or even similar) movements may expect to see some increases as well; however, these increases will be of a lesser magnitude compared to the primary strength assessment. Future research should begin to quantify how much generality exists amongst various increases in strength, particularly as it related to skills in the weight room and sports specific movements/skills.

\section{REFERENCES}

1. Rasch PJ, Morehouse LE. Effect of static and dynamic exercises on muscular strength and hypertrophy. J Appl Physiol 1957;11:29-34.

2. Dankel SJ, Kang M, Abe T et al. Resistance training induced changes in strength and specific force at the fiber and whole muscle level: a metaanalysis. Eur J Appl Physiol 2019;119:265-278

3. Chin ER, Olson EN, Richardson JA et al. A calcineurin-dependent transcriptional pathway controls skeletal muscle fiber type. Genes Dev 1998;12;16:2499-2509

4. Westerblad H, Allen D. Changes of myoplasmic calcium concentration during fatigue in single mouse muscle fibers. J Gen Physiol 1991;98:615635 .

5. Canepari M, Rossi R, Pellegrino M et al. Effects of resistance training on myosin function studied by the in vitro motility assay in young and older men. J Appl Physiol 2005;98:2390-2395.

6. Mattocks KT, Buckner SL, Jessee MB et al. Practicing the Test Produces Strength Equivalent to Higher Volume Training. Med Sci Sports Exerc 2017;49:1945-1954.

7. Dankel SJ, Counts BR, Barnett BE et al. Muscle adaptations following 21 consecutive days of strength test familiarization compared with traditional training. Muscle Nerve 2017;56:307-314.

8. Buckner SL, Jessee MB, Mattocks KT et al. Determining Strength: A Case for Multiple Methods of Measurement. Sports Med 2017;47:193-195.

9. Gentil P, Del Vecchio FB, Paoli A et al. Isokinetic Dynamometry and 1RM Tests Produce Conflicting Results for Assessing Alterations in Muscle Strength. J Hum Kinet 2017;56:19-27.

10. Hortobagyi T, Katch FI, LaChance PF. Interrelationships among various measures of upper body strength assessed by different contraction modes. Eur J Appl Physiol Occup Physiol 1989;58:749-755.

11. Rasch PJ. Relationship between maximum isometric tension and maximum isotonic elbow flexion. Res $Q$ 1957;28:85-85.

12. Rasch PJ, Pierson WR. Relationship between maximum isometric tension and breaking strength of forearm flexors. Res $Q$ 1960;31:534-535.

13. Mitchell CJ, Churchward-Venne TA, West DW et al. Resistance exercise load does not determine training-mediated hypertrophic gains in young men. J Appl Physiol 2012;113:71-77.

14. Ogasawara R, Loenneke JP, Thiebaud RS et al. Low-load bench press training to fatigue results in muscle hypertrophy similar to high-load bench press training. Int J Clin Med 2013;4:114.

15. Martin-Hernandez J, Marin PJ, Menendez H et al. Muscular adaptations after two different volumes of blood flow-restricted training. Scand J Med Sci Sports 2013;23:e114-120.

16. Ward J, Fisk GH. The Difference in Response of the Quadriceps and the Biceps Brachii Muscles to Isometric and Isotonic Exercise. Arch Phys Med Rehabil 1964;45:614-620.

17. Fisher JP, Steele J. Heavier and lighter load resistance training to momentary failure produce similar increases in strength with differing degrees of discomfort. Muscle Nerve 2017;56:797-803.

18. Fisher JP, Stuart C, Steele J et al. Heavier- and lighter-load isolated lumbar extension resistance training produce similar strength increases, but different perceptual responses, in healthy males and females. PeerJ 2018;6:e6001.

19. Moritani T, deVries HA. Neural factors versus hypertrophy in the time course of muscle strength gain. Am J Phys Med 1979;58:115-130.

20. Loenneke JP, Wilson JM, Marin PJ et al. Low intensity blood flow restriction training: a meta-analysis. Eur J Appl Physiol 2012;112:18491859.

21. Morton RW, Oikawa SY, Wavell CG et al. Neither load nor systemic hormones determine resistance training-mediated hypertrophy or strength gains in resistance-trained young men. $J$ Appl Physiol 2016;121:129-138.

22. Sale D, Martin J, Moroz D. Hypertrophy without increased isometric strength after weight training. Eur J Appl Physiol Occup Physiol 1992;64:51-55.

23. Rutherford OM, Greig CA, Sargeant AJ et al. Strength training and power output: transference effects in the human quadriceps muscle. J Sports Sci 1986;4:101-107.

24. Fisher J, Bruce-Low S, Smith D. A randomized trial to consider the effect of Romanian deadlift exercise on the development of lumbar extension strength. Phys Ther Sport 2013;14:139-145.

25. Jessee MB, Buckner SL, Mouser JG et al. Muscle adaptations to high-load training and very low-load training with and without blood flow restriction. Front physiol 2018;9:1448 